\title{
Revisiting the role of introgression vs shared ancestral polymorphisms as key processes shaping genetic diversity in the recently separated sibling species of the Anopheles gambiae complex
}

\author{
MJ Donnelly, ${ }^{1,2}$, J Pinto ${ }^{3}$, R Girod ${ }^{4}$, NJ Besansky ${ }^{5}$ and T Lehmann ${ }^{2}$ \\ ${ }^{1}$ Vector Research Group, Liverpool School of Tropical Medicine, Pembroke Place, Liverpool L3 5QA, UK; ${ }^{2}$ Centers for Disease Control and \\ Prevention, MS F22, 4770, Buford Hwy, Chamblee, GA 30341, USA; ${ }^{3}$ Centro de Malaria e outras Doencas Tropicais/Instituto de Higiene \\ e Medicina Tropical, Rua da Junqueira 96, 1349-008 Lisbon, Portugal; ${ }^{4}$ Mission de Prévention et de Lutte contre les Endémies \\ Vectorielles, DRASS, BP 9, 97408 St Denis, La Réunion, France; ${ }^{5}$ Department of Biological Sciences, University of Notre Dame, Notre \\ Dame, IN 46556, USA
}

The role of interspecific hybridisation in the evolution of pest species is poorly understood. In mosquito disease vectors this is of particular importance due to the evolution of insecticide resistance and the proposed release of transgenic strains that are refractory to the malaria parasite. In this study, we apply population genetic methods in a novel manner to determine whether mitochondrial DNA sequences have introgressed between the closely related African malaria vectors Anopheles gambiae and A. arabiensis. Our results suggest that speciation was geologically recent and ancestral haplotypes at the ND5 locus are retained in both species. In addition, comparing haplotype frequencies in allopatric and sympatric populations, suggest locale specific unidirectional introgression of mitochondria from $A$. arabiensis into $A$. gambiae.

Heredity (2004) 92, 61-68, advance online publication, 3 December 2003; doi:10.1038/sj.hdy.6800377

Keywords: hybridisation; speciation; disease vectors; malaria; filariasis

\section{Introduction}

A major question in evolutionary biology is whether interspecific hybridisation is a significant route for the transfer of genetic adaptations (Barton, 2001). Most studies of introgression have focused on plant systems (Martinsen et al, 2001). Where animal studies have been performed they have been in tractable systems such as hybrid zones (Evans et al, 2001), where species are morphologically distinguishable (Beaumont et al, 2001) or where exotic species have been introduced (Goodman et al, 1999). In these studies, the organisms have evolved separately for a long period and introgressed alleles can be readily identified due to mutational differences or differing allele distributions (Goodman et al, 1999; Roques et al, 2001).

The Anopheles gambiae complex of mosquito species forms the most important insect disease vector system. Of the seven recognised species within the complex, A. gambiae s.s. and A. arabiensis are the most abundant and widespread, occurring in sympatry over most of their distributions. In sub-Saharan Africa they are the primary malaria vectors, the main vectors of Bancroftian filariasis in rural areas and have localised importance in arbovirus transmission. In these and many other disease

Correspondence: MJ Donnelly, Vector Research Group, Liverpool School of Tropical Medicine, Pembroke Place, Liverpool L3 5QA, UK.

E-mail:mjames@liv.ac.uk

Received 21 January 2003; accepted 10 July 2003 vector complexes, it is difficult to identify introgressed alleles, possibly because the vectors have only recently speciated and there has been insufficient time to accumulate mutations (Coluzzi, 1982; Powell et al, 1999; Walton et al, 2000). Hybrids occur naturally in the wild, in some regions at frequencies of up to 2 per 1000 (White et al, 1972; Tripet et al, 2001) but the evolutionary significance of these hybrids is unclear. It is not known if genes are actually introgressed into parent species by backcrossing of fertile F1 hybrid females (males are sterile). In laboratory experiments polymorphic chromosomal inversions can be transferred between species (della Torre et al, 1997). Some workers have postulated that certain chromosomal inversions, that are associated with aridity tolerance, may have introgressed from $A$. arabiensis into $A$. gambiae and have enabled this species to spread into novel habitats (Powell et al, 1999).

Phylogenetic approaches to resolving taxa relationships in these species have been hampered by high intraspecific and low interspecific variation (Besansky et al, 1994; Krzywinski et al, 2001). Previous studies of partial sequence of mitochondrial DNA have shown that large numbers of haplotypes are shared between A. gambiae, A. arabiensis and A. bwambae (Besansky et al, 1997; Thelwell et al, 2000; Donnelly et al, 2001), but whether this is a result of introgression or retention of ancestral polymorphisms is unresolved (Besansky et al, 1997; Thelwell et al, 2000). In this study, we examine patterns of intra and interspecific differentiation in ways that can allow us to distinguish between contemporary 
introgression and retention of ancestral mitochondrial sequences. We propose that if introgression is ongoing, $F_{\mathrm{ST}}$ values between sympatric populations will be lower than between interspecific allopatric comparisons. Furthermore, this approach can reveal locale specific introgression and can determine if introgression occurs in a unidirectional or bidirectional manner.

The data in this study also serve as additional markers to compare with the large number of microsatellite-based studies that have investigated population structuring in these organisms on micro- and macrogeographic scales (Lehmann et al, 1997, 2003; Kamau et al, 1998; Lanzaro et al, 1998; Simard et al, 1999; Donnelly and Townson, 2000; Wondji et al, 2002).

\section{Materials and methods}

Mosquitoes were collected from 25 sites throughout subSaharan Africa (Figure 1 and Table 1). Most locations have been described in detail previously (Besansky et al, 1997; Lehmann et al, 1997, 1998, 2003; Donnelly and Townson, 2000; Pinto et al, 2002). Species identification, collection location and sample size are given in Table 1. Adult mosquitoes were obtained from houses within a village by resting and pyrethrum knockdown collections. A sample consisted of specimens collected in an area with a radius less than $1 \mathrm{~km}$. Previous studies revealed no population subdivision within and among adjacent villages separated by $10-50 \mathrm{~km}$ and that mosquitoes within a house represent a random sample of the population (Petrarca and Beier, 1992; Besansky et al, 1997; Lehmann et al, 1997; Donnelly et al, 1999). To maximise the power of interspecific analyses specimens were pooled from the three regions where $A$. gambiae and A. arabiensis occur in sympatry, Kenya, Senegal and Malawi.

Recent work suggests that there are two forms of uncertain taxonomic status within A.gambiae, which may be characterised by their rDNA molecular type (Favia et al, 1997; Wondji et al, 2002). All the samples used in this study are the $S$ rDNA type except samples from Senegal and Ghana, which are from populations that only exhibit the $M$ form (Lehmann et al, 2003) and the sample from Sao Tome and Principe which is also $\mathrm{M}$ form (Pinto et al, 2003). There is no evidence for reproductively isolated forms within $A$. arabiensis. After DNA extraction (Lehmann et al, 1997; Donnelly et al, 1999) and PCR-based species identification (Scott et al, 1993) individuals were sequenced over a $650 \mathrm{bp}$ stretch of the ND5 region of the mitochondrial genome following the protocols of $\mathrm{Be}-$ sansky et al (1997). DNA sequencing was performed on ABI 377 or ABI 3100 machines (Perkin-Elmer) using fluorescent labelling technology and standard analytical protocols. Sequences were unambiguously aligned using the Clustal W option in Sequence Navigator (Version 1.0.1; ABI Systems, CA, USA).

\section{$F_{\mathrm{ST}}$-based methods}

We estimated intra- and interspecific differentiation between samples using $F_{\mathrm{ST}}$ (Hudson et al, 1992). A weighted means approach was used to account for differences in sample size. Significance of $F_{\mathrm{ST}}$ values was estimated using a permutation program written in the SAS language with 2000 replications (SAS Institute, 1990). Pairwise estimates of $F_{\mathrm{ST}}$ were used as distance measures to generate a neighbour-joining (NJ) tree using Mega V2.1 (Kumar et al, 2001).

\section{Phylogeography}

A haplotype tree was constructed using the statistical parsimony algorithm of Templeton et al (1992). The TCS 1.13 program of Clement et al (2000) was used to estimate the haplotype tree.

\section{Results}

Complete and unambiguous sequence of a $650 \mathrm{bp}$ section of the ND5 region of the mitochondrial genome was obtained for a total of 331 specimens (Table 1). In addition to newly generated sequences of $52 \mathrm{~A}$. gambiae and 61 A. arabiensis specimens, we used data from 68 A. gambiae and 50 A. arabiensis published by Besansky et al (1997) and 45 A. gambiae published by Lehmann et al (1997) and 55 A. arabiensis for which summary statistics were published previously (Donnelly et al, 2001). Reference sequences have been deposited in Genbank (accession numbers AY312090-AY312142). No nuclear/ nonfunctional copies of the gene were present as evidenced by the very low pairwise divergence, the absence of stop codons, the predominance of synonymous substitutions and the unambiguous electropherograms.

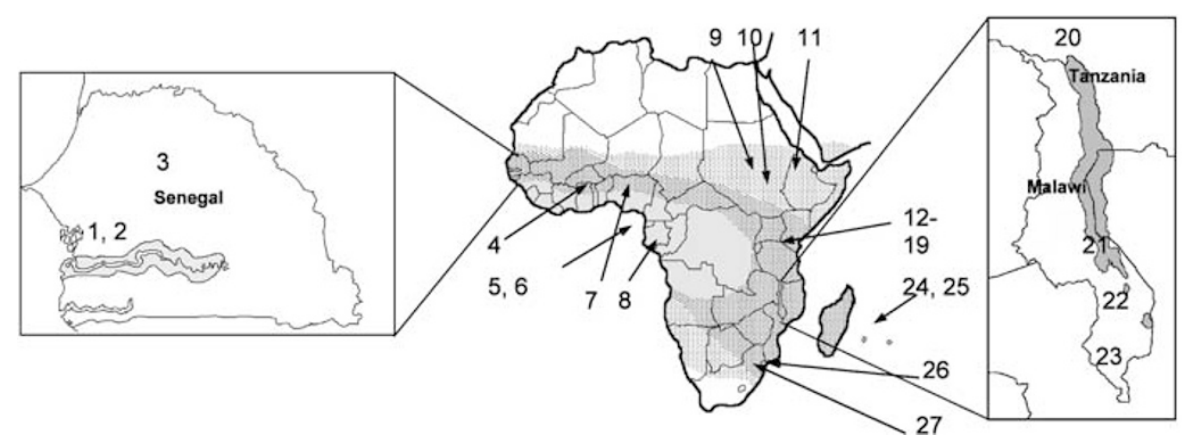

Figure 1 Location of sample sites. Species ranges are marked for A. arabiensis (stippled) and for A. gambiae (grey). More detailed information for sampling locations 12-19 can be found in Besansky et al (1997). The key to locations is indicated by the location superscripts in Table 1. 
Table 1 Geographic distribution and details of ND5 haplotype for each sample

\begin{tabular}{|c|c|c|c|c|}
\hline \multirow[t]{2}{*}{ Location } & \multicolumn{2}{|r|}{ Anopheles gambiae } & \multicolumn{2}{|c|}{ Anopheles arabiensis } \\
\hline & Sample size & Haplotypes & Sample size & Haplotypes \\
\hline \multicolumn{5}{|l|}{ Senegal } \\
\hline Dielmo $(\mathrm{M})^{1}$ & $10^{\mathrm{a}}$ & $2(4), 3(2), 4,16,35,39$ & $9^{a}$ & $\begin{array}{l}5,1718,19,20,21 \\
22,23,26\end{array}$ \\
\hline Ndiop $(\mathrm{M})^{2}$ & $9^{\mathrm{a}}$ & $2,3(2), 6,7,24,25,27,34$ & $9^{a}$ & $\begin{array}{l}\mathbf{1}(2), 10, \mathbf{1 1}, 12,13 \\
\mathbf{1 4}, 15, \mathbf{3 7}\end{array}$ \\
\hline Barkedji $(\mathrm{M})^{3}$ & $9^{\mathrm{a}}+4$ & $2,3(5), 9,28,29,33(2), 38,106$ & $8^{\mathrm{a}}$ & $\begin{array}{l}1,2,8,33(2), 34,35 \\
36\end{array}$ \\
\hline \multicolumn{5}{|l|}{ Ghana } \\
\hline Navrongo $(\mathrm{M})^{4}$ & 6 & $3(4), 33,52$ & & \\
\hline \multicolumn{5}{|l|}{ Sao Tome and Principe } \\
\hline Praia Burras $(\mathrm{M})^{5}$ & 2 & $79(2)$ & & \\
\hline Porto Allegre $(\mathrm{M})^{6}$ & 1 & 3 & & \\
\hline \multicolumn{5}{|l|}{ Nigeria } \\
\hline Gwamlar (S) & 9 & 14, 33(2), 59, 63, 64, 89, 91, 104 & 2 & 1,2 \\
\hline \multicolumn{5}{|c|}{ 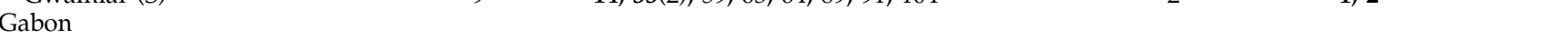 } \\
\hline $\operatorname{Dienga}(S)^{8}$ & 2 & 11,32 & & \\
\hline \multicolumn{5}{|l|}{ Sudan } \\
\hline Wad Awad ${ }^{9}$ & & & 9 & $\begin{array}{l}\text { 1, 14, 20, 33, 41, 71, } \\
85,92,96,\end{array}$ \\
\hline Um Rakuba ${ }^{10}$ & & & 13 & $\begin{array}{l}\mathbf{1}(5), 5, \mathbf{1 1}(2), 60,66, \\
70,88,105\end{array}$ \\
\hline \multicolumn{5}{|l|}{ Ethiopia } \\
\hline Harosha $^{11}$ & & & $28^{\mathrm{b}}$ & $\begin{array}{l}\mathbf{1}(14), \mathbf{1 1}(7), \mathbf{3 3}(2) \\
\mathbf{4 1}, 61,72,84(2)\end{array}$ \\
\hline \multicolumn{5}{|l|}{ Kenya } \\
\hline Asembo $(S)^{12}$ & $45^{\mathrm{b}}$ & $\begin{array}{l}\text { 1(2), 2(3), 3, 14(2), 33(8), 35, 36, 37, } \\
48(2), 49,52,56,59(5), 62,63,65(2) \\
67,68,78,80,81,87,90,94(2), 95,102 \\
103\end{array}$ & 15 & $\begin{array}{l}\text { 1(5), 11(5), 33, 41(3), } \\
77,\end{array}$ \\
\hline Escarpment $(S)^{13, d}$ & $6^{\mathrm{a}}$ & $33,42,50,51,52,53$ & $2^{\mathrm{a}}$ & 1,41 \\
\hline Ahero $(S)^{14}$ & & & $4^{\mathrm{a}}$ & $1,11,42,43$ \\
\hline Kisian $(S)^{15}$ & $4^{\mathrm{a}}$ & $2,11,32,54$ & $2^{\mathrm{a}}$ & 1,44 \\
\hline Wathrego $(S)^{16}$ & $6^{a}+11^{c}$ & $\begin{array}{l}\mathbf{2}, 11,33(5), 48,52,53,107,108,109 \\
110111,112,113\end{array}$ & $5^{\mathrm{a}}$ & $1(2), 11,43,45$ \\
\hline Nyakoch $(S)^{17}$ & $4^{\mathrm{a}}$ & $\mathbf{2}, \mathbf{1 1}, 55,56$ & & \\
\hline Muhroni (S) ${ }^{18}$ & $4^{a}$ & $33(2), 58,59$ & $4^{\mathrm{a}}$ & $1,2,41,46$ \\
\hline Jego $(S)^{19}$ & $5^{\mathrm{a}}$ & $32(2), 41(2), 57$ & $2^{\mathrm{a}}$ & $1(2)$ \\
\hline \multicolumn{5}{|l|}{ Tanzania } \\
\hline Kyela $(S)^{20}$ & 9 & $1(2), 11,32,37,41,54,82,83$ & & \\
\hline \multicolumn{5}{|l|}{ Malawi } \\
\hline Mkali $(S)^{21}$ & 2 & 32,37 & $27^{\mathrm{b}}$ & $\begin{array}{l}\mathbf{1}, \mathbf{2}, \mathbf{1 1}(2), 30, \mathbf{3 2}(7) \\
\mathbf{4 3}, 44,45(5), \mathbf{5 4}(4) \\
73,74,99, \mathbf{1 0 0}\end{array}$ \\
\hline Thyolo (S) ${ }^{22}$ & 16 & $\begin{array}{l}\mathbf{1}, \mathbf{1 1}(2), \mathbf{3 1}, \mathbf{3 2}(5), \mathbf{4 3}, 75,83,93,98(2) \\
\mathbf{1 0 0}\end{array}$ & & \\
\hline Seseo $(S)^{23}$ & 1 & 76 & 13 & $\begin{array}{l}30, \mathbf{3 1}, \mathbf{4 3}, 45(2), \mathbf{5 0}, \\
54(2), 69,86(2), 97, \\
101\end{array}$ \\
\hline \multicolumn{5}{|l|}{ Reunion } \\
\hline Riv. des Galets ${ }^{24}$ & & & 1 & 11 \\
\hline Riv. du Mat ${ }^{25}$ & & & 5 & 11(5) \\
\hline \multicolumn{5}{|l|}{ Mozambique } \\
\hline Maputo $^{26}$ & & & 3 & 11(3) \\
\hline \multicolumn{5}{|l|}{ South Africa } \\
\hline Malahlapanga $^{27}$ & & & $5^{\mathrm{a}}$ & 11, 30(3), 32 \\
\hline
\end{tabular}

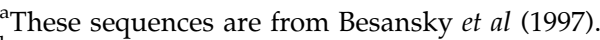

${ }^{\mathrm{b}}$ Summary statistics for these sequences were previously published in Donnelly et al (2001).

${ }^{\mathrm{c}}$ These sequences are from Lehmann et al (1997).

${ }^{\mathrm{d}}$ Refers to whether the samples are from an $\mathrm{M}$ or $\mathrm{S}$ form population of A. gambiae.

Superscript numbers refer to the sampling locations detailed in Figure 1. Haplotype numbers are the same as stated in Besansky et al (1997) up to haplotype 59. Haplotypes marked in bold are shared between both species. Figures in brackets are frequencies for each haplotype. 
Intraspecific estimates of differentiation

A. arabiensis: Pairwise estimates of differentiation as inferred from $F_{\mathrm{ST}}$ statistics were generally low (Figure 2a; mean $\pm S E=0.098 \pm 0.027$; Data matrix available from authors). Strong evidence for structuring was observed between the sample from the island of Reunion and continental populations. Only a single haplotype was present in the Reunion sample $(n=6)$ probably reflecting a lower effective population size $\left(N_{\mathrm{e}}\right)$ on the island. Therefore, as the lower $N_{\mathrm{e}}$ will complicate interpretation of differentiation this sample was excluded from the analysis. Remaining pairwise $F_{\mathrm{ST}}$ values were used to construct an NJ tree (Figure 2a). Three clusters were observed: Sudan and Senegal (cluster 1); Western Kenya and Ethiopia (cluster 2) and Malawi (cluster 3).

a

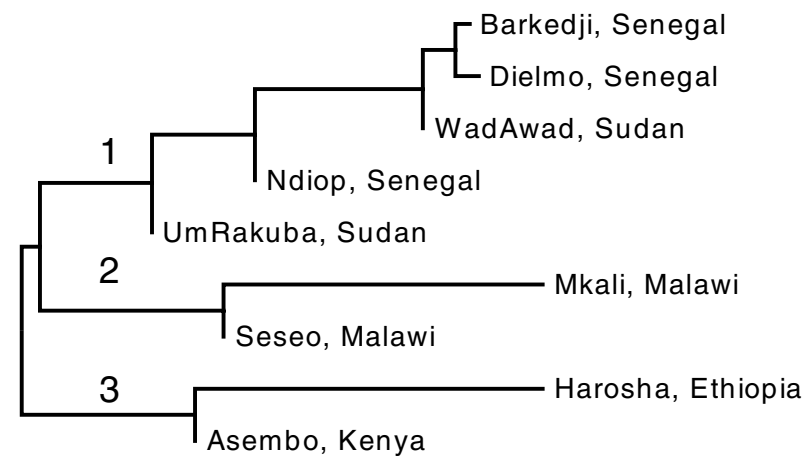

0.02

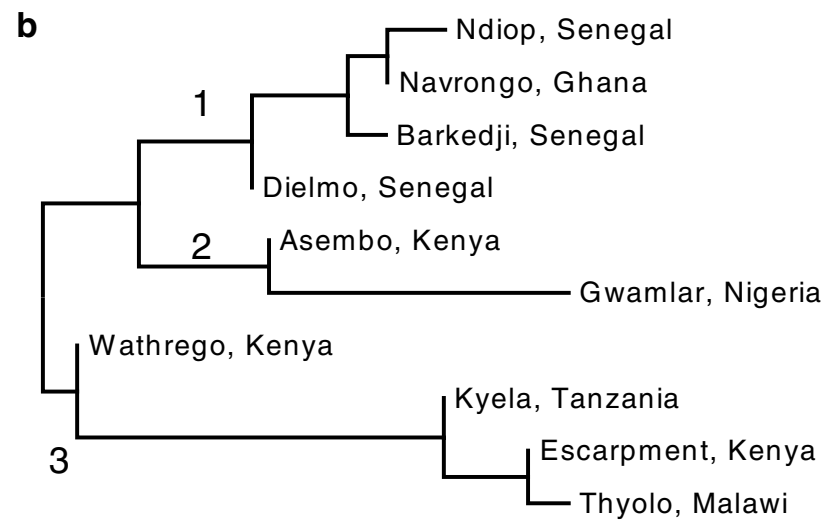

Figure $2 F_{\mathrm{ST}}$ distance-based trees for $A$. arabiensis (a) and A. gambiae (b) recovered from partial $650 \mathrm{bp}$ ND5 mitochondrial DNA sequences. The trees were constructed using an NJ method based upon a pairwise interpopulation values of $F_{\mathrm{ST}}$. Branch lengths (see scale bar) are proportional to the mean pairwise $F_{\mathrm{ST}}$ values. For the A. arabiensis data there were no significant pairwise comparisons of $F_{\mathrm{ST}}$ within each of the clusters $(P>0.05)$. When samples from different clusters were compared, $F_{\mathrm{ST}}$ values were significantly different $(P<0.05)$ for $30 \%$ (clusters 1 and 3 ), 40\% (clusters 1 and 2 ) or $50 \%$ (clusters 2 and 3 ) of pairwise comparisons. For the A. gambiae data when samples from different clusters were compared, $F_{\mathrm{ST}}$ values were significantly different $(P<0.05)$ for $56 \%$ (clusters 1 and 3), 62\% (clusters 1 and 2) or 50\% (clusters 2 and 3) of pairwise comparisons.

A. gambiae: The NJ tree suggested a primary division between samples from West African/western Kenya (clusters 1 and 2) with samples from Tanzania and Malawi (cluster 3) (Figure 2b). The genetic division corresponds with the topographic division of populations by the Rift Valley complex. Ghanaian and Senegalese populations grouped closely (cluster 1), while Nigerian specimens grouped more closely with populations from Western Kenya (cluster 2). These groupings run contrary to the geographical distance. Notably, cluster 1 comprises $M$ form specimens whereas $S$ form specimens are found in clusters 2 and 3. There was only one significant pair wise comparison of $F_{\mathrm{ST}}$ within the clusters (cluster 3 Thyolo-Wathrego) but over half of the pairwise comparisons between clusters were significant (Figure 2). The Wathrego sample and the Escarpment sample are both from Western Kenya and the grouping with Tanzanian and Malawian samples from east of the Rift Valley Complex is likely to be a result of the lower sample size of these Kenyan samples (Lehmann et al, 2000). Pairwise values of $F_{\mathrm{ST}}$ were high for comparisons involving Wathrego and Escarpment but resampling tests were insignificant (data matrix available from authors).

\section{Haplotype networks}

For these data haplotypes separated by up to 11 mutational steps have a probability of $\geqslant 0.95$ of being connected in a parsimonious manner. The statistical parsimony algorithm produced networks for both species within the 11-step limit of parsimony but there were a number of ambiguities within the tree, apparently due to homoplasy within the mitochondrial sequences. These ambiguities were resolved following the suggestions of Templeton et al (1992) and Crandall and Templeton (1993) (Figures 3 and 4). In general this resulted in the haplotype being placed externally and linked to one of the high-frequency internal alleles. The high levels of homoplasy observed in our data are common in Anopheles (Walton et al, 2000). At present the evidence suggests that the homoplasy reflects mutational hot spots rather than recombination. There was no evidence for heteroplasmy in our electropherograms and of the 72 polymorphic sites within the data set there were 10 sites with three variants and one site with four variants suggesting large variation in mutation rates across the locus.

\section{Introgression and ancestral retention}

There were no fixed nucleotide differences between the species and 17 out of 113 haplotypes were shared between species (Table 1). Mean $F_{\mathrm{ST}}( \pm 95 \%$ CI) for sympatric $(n=3)$ and allopatric $(n=6)$ comparisons were $0.070( \pm 0.104)$ and $0.121( \pm 0.068)$, respectively (Table 2$)$ $(P>0.05)$. While estimates of $F_{\mathrm{ST}}$ involving $A$. gambiae from Malawi were nonsignificant $(P>0.05)$ the two allopatric comparisons involving $A$. arabiensis from the same sample locations were highly significant $(P<0.001)$ (Table 2).

Of the shared haplotypes only two (Nos. 2 and 33 Table 1 ) were common to $A$. arabiensis and $A$. gambiae $\mathrm{M}$ and $S$ forms, and were internal to both species haplotype trees (Figures 3 and 4). The remaining shared haplotypes were found predominantly in $A$. arabiensis and $S$ form 


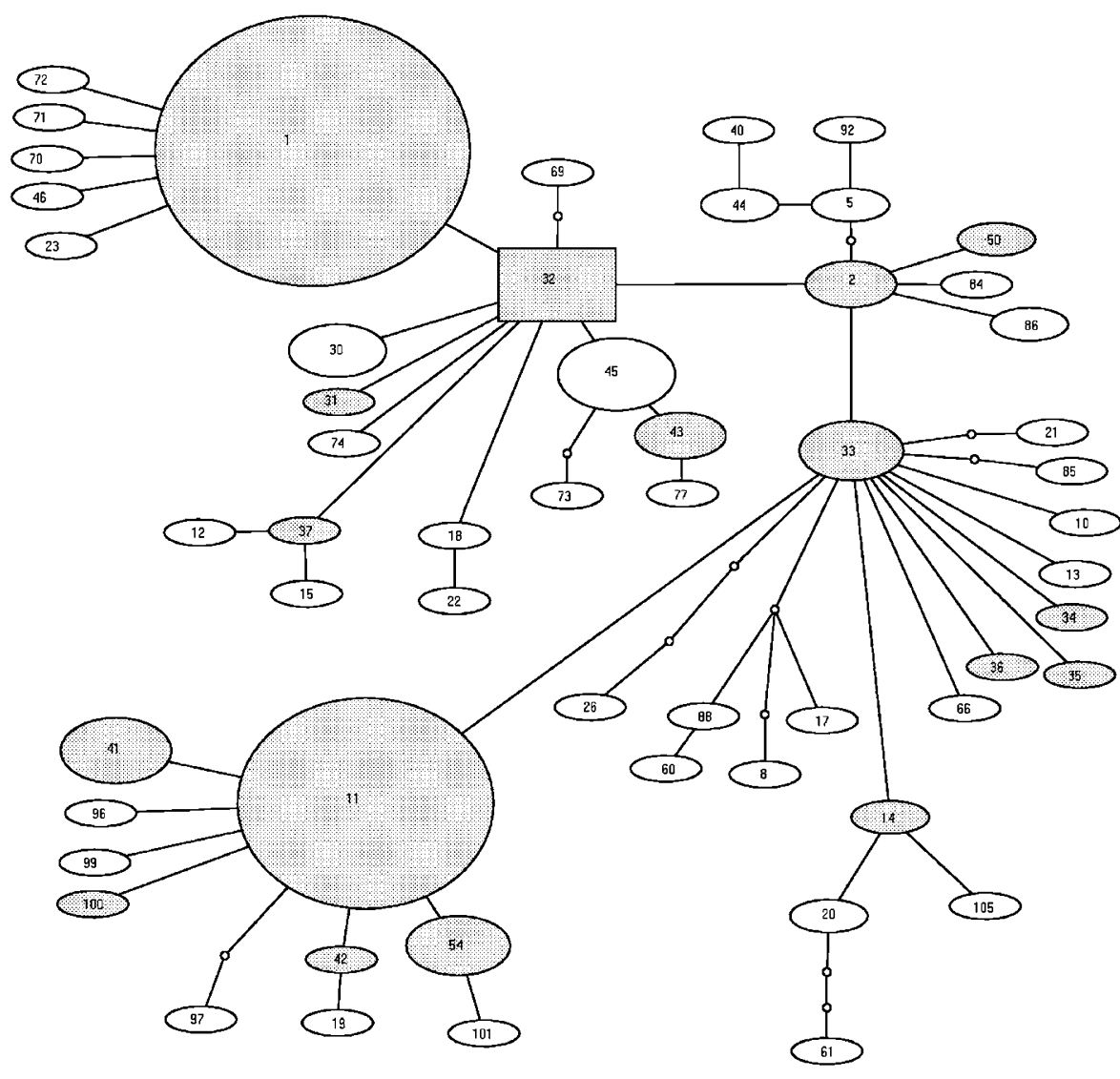

Figure 3 Statistical parsimony network for A. arabiensis data set. Haplotype node area is proportional to the number of specimens contained. Links between nodes are all single mutational steps regardless of length. Shaded nodes are haplotypes that are found in both species.

comparisons $(n=11)$ rather than between $A$. arabiensis and $\mathrm{M}$ form comparisons $(n=2)$. The shared haplotypes are found throughout the species distribution of A. gambiae although there is an apparent clustering of shared haplotypes in certain populations. Kyela, Tanzania; Mkali, Malawi; Kisian, Kenya and Dienga, Gabon. Five shared haplotypes were found in samples of A. arabiensis from Ethiopia, Sudan and Reunion (Table 1). A. gambiae is absent from the samples collected from these countries and therefore the presence of these haplotypes must reflect noncontemporary processes such as incomplete lineage sorting between the two species or historical introgression events. As would be predicted if ancestral polymorphisms were retained, the majority of shared haplotypes in these populations (four of five; Figure 3 and Table 1) were internal to the network. Similarly in the single species collection from Gabon, where A. arabiensis is absent, there were also shared haplotypes, again internal to the haplotype network (Figure 4 and Table 1) and reflecting a noncontemporary process. In $A$. gambiae except for two populations the proportion and frequency of haplotypes that were common to both species was between 22-50 and 17-50\%, respectively. However, in samples from Tanzania and Malawi these figures rose to 75-78 and 67-68\%, respectively.

\section{Discussion}

These data provide a corollary of the results of microsatellite-based macrogeographic studies of differentia- tion in both species (Lehmann et al, 1997, 2003; Kamau et al, 1998; Lanzaro et al, 1998; Simard et al, 1999; Donnelly and Townson, 2000). In general, as in microsatellite-based studies the levels of population differentiation were lower in A. arabiensis (Figure 2) (Donnelly and Townson, 2000; Lehmann et al, 2003) and the only large estimates of $F_{\mathrm{ST}}$ involved an island population which is likely to have experienced founding effects / genetic bottleneck (Simard et al, 1999; Donnelly et al, 2002). Surprisingly, samples of $A$. arabiensis from Senegal and Sudan were grouped in a cluster, which runs contrary to geographic distance. Similar patterns have been observed in $A$. gambiae and were thought to reflect similarities in ecological zones and the absence of topographic barriers to gene flow such as those that may isolate Sudanese samples from those to the south (Lehmann et al, 2003).

In $A$. gambiae there was a clear distinction between populations of $\mathrm{M}$ and S rDNA forms and within the Sform comparisons between samples from the West and East of the Rift Valley complex. These data are in accordance with the studies of Wondji et al (2002) and Lehmann et al (2003) that provided evidence for a degree of genetic isolation between $M$ and $S$ forms. The failure of other studies to detect differences between $M$ and $S$ forms reflects how recent the disruption of gene flow must have been (Gentile et al, 2001) or conversely that ongoing gene flow may be homogenising allele arrays in both forms, outside certain regions of the genome. This study utilised haplotype frequency-based approaches, 


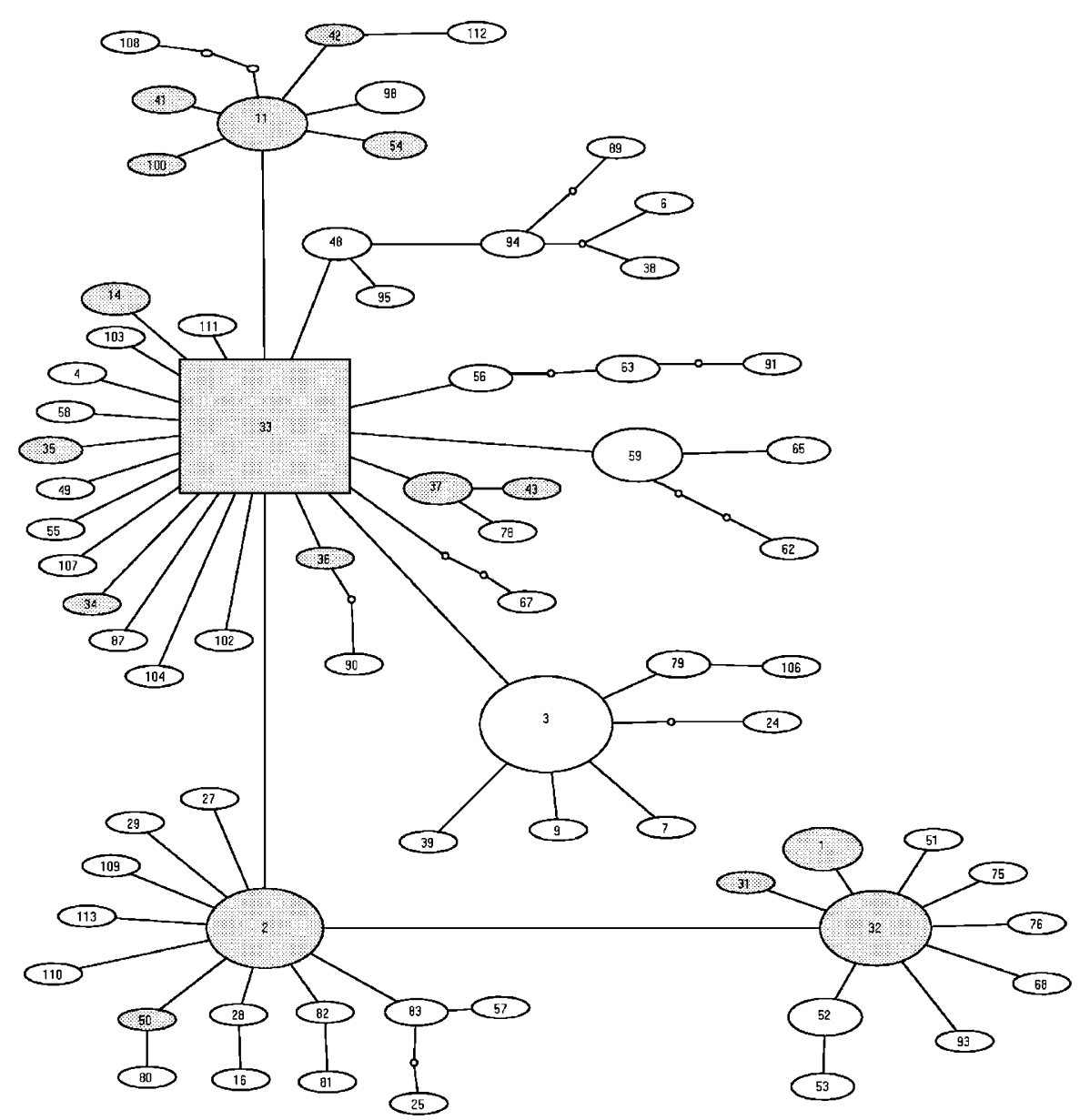

Figure 4 Statistical parsimony network for A. gambiae data set. Haplotype node area is proportional to the number of specimens contained. Links between nodes are all single mutational steps regardless of length. Shaded nodes are haplotypes that are found in both species.

which are more sensitive to more recent separation events since they are not reliant upon the accumulation of infrequent mutation events.

\section{Introgression and ancestral retention}

When contemporary introgression can be discounted, due to absence of one of the species from a locale, there are large numbers of haplotypes shared between species. Whether these shared sequences are true ancestral retentions or traces of recent introgression events cannot be determined and caution against definitive statements. These data suggest that for closely related taxa even very extensive mitochondrial DNA data sets may have insufficient power to conclusively resolve between the conflicting hypotheses of ancestral retention and contemporary introgression in certain populations.

Mitochondrial introgression is the most parsimonius explanation for the similarity in haplotype arrays between some sympatric populations of $A$. gambiae and $A$. arabiensis but introgression is apparently not an ubiquitous phenomenon. If introgression was occurring between all sympatric populations of $A$. gambiae and $A$. arabiensis then we would expect interspecific estimates of $F_{\mathrm{ST}}$ to be significantly lower in sympatric rather than allopatric comparisons. This was not observed in these data. However, interspecific $F_{\mathrm{ST}}$ analyses involving
A. gambiae from Malawi were all nonsignificant whereas those interspecific comparisons involving Malawian $A$. arabiensis and A. gambiae from Kenya and Senegal were both highly significant (Table 2). This suggests that, since the haplotype distributions in Malawian A. gambiae are similar to all three $A$. arabiensis distributions, introgression in Malawi is likely to be a unidirectional process from $A$. arabiensis into $A$. gambiae. However, it should be noted that sample size was lowest in the sample of A. gambiae from Malawi and that mtDNAbased phylogenies using colonised specimens of A. gambiae and A. arabiensis suggested that introgression may have occurred in the opposite direction (Caccone et al, 1996). However, these two species have a far wider species distribution than other members of the complex. This is likely to result in higher effective population size and therefore since genetic drift will be lower there may well be a greater retention of ancestral haplotypes in these species than in other members of the complex, despite the possible closer phylogenetic proximity of different species pairs. There were insufficient data to apply similar tests to samples from Tanzania but a large number and proportion of haplotypes were shared between sympatric populations in Tanzania suggesting that introgression may be occurring in this region as well. A. arabiensis occurs at much higher frequencies than A. gambiae in the study sites in Tanzania and Malawi 
Table 2 Interspecific pairwise estimates of $F_{\mathrm{ST}}$

\begin{tabular}{lllll}
\hline & & \multicolumn{3}{c}{ A. arabiensis } \\
\cline { 3 - 5 } & & Kenya (34) & Malawi (40) & Senegal (26) \\
\hline \multirow{2}{*}{ A. gambiae } & Kenya (85) & 0.1318 & 0.1398 & 0.0760 \\
& Malawi (19) & 0.0318 NS & -0.0356 NS & 0.0421 NS \\
& Senegal (32) & 0.2309 & 0.2061 & 0.1137 \\
\hline
\end{tabular}

$F_{\mathrm{ST}}$ calculated using the formulas of Hudson et al (1992) with weighting for population size. Significance of $F_{\mathrm{ST}}$ estimates was evaluated against the results from 2000 random permutations executed by a program written in the SAS language (SAS Institutes, 1990). Italic numerals indicate $P<0.001$. NS, not significant.

(Charlwood et al, 2000; Spiers et al, 2002) and differing species' density is thought to be one of the major determinants of introgression (Avise and Saunders, 1984). Whether introgression is frequent enough to play a role in the differentiation within $A$. gambiae $S$ form that is observed either side of the Rift Valley remains to be investigated.

An analysis of the relative position of shared haplotypes on the species networks, based on the assumption that on average the haplotypes found interior to the haplotype network should be older than those found at the tips, was also suggestive of ongoing introgression. If there is contemporary introgression both old and more recently derived haplotypes are equally likely to cross the species barrier and therefore shared haplotypes will not preferentially occur at internal nodes. An exact test based upon the location (internal/external) of shared haplotypes in each species tree also showed no evidence for significant differences $(P>0.05)$. This approach, although far from conclusive for our data given the number of ambiguities within the species trees, may be a powerful way of detecting introgression in those species with more robust and deeper networks.

Recent studies have demonstrated the importance of unidirectional introgression events in evolution associated with environmental changes (Grant and Grant, 2002). In the A.gambiae complex the interspecific transfer of DNA we observed at mitochondrial loci is likely occur at nuclear loci (Besansky et al, 2003) but it is unknown if this process is important for the acquisition of selectively advantageous genes. However, the evidence for introgression in natural populations and, in particular, from $A$. arabiensis into $A$. gambiae, lends credence to the hypothesis of Powell et al (1999) that some selectively advantageous genes may have moved from $A$. arabiensis into A. gambiae. Furthermore, while A. gambiae has been thought to be undergoing incipient speciation in certain locations the converse may actually be occurring and the $\mathrm{M}$ and $\mathrm{S}$ forms and $A$. arabiensis may be converging to form a hybrid swarm. This is of particular concern given widespread insecticide resistance and possibilities of transgenic release in these highly pernicious malaria vectors.

\section{Acknowledgements}

MJD was supported by a post-doctoral fellowship from the American Society of Microbiology. This work received financial support from the UNDP/World Bank/WHO Special Programme for Research and Training in Tropical Diseases and from the NIH Grant
(A140631-01). JP was funded by Praxis XXI/FCT (BD/ 15754/98).

\section{References}

Avise JC, Saunders NC (1984). Hybridization and introgression among species of sunfish (Lepomis): analysis by mitochondrial DNA and allozyme markers. Genetics 108: 237-255.

Barton NH (2001). The role of hybridization in evolution. Mol Ecol 10: 551-568.

Beaumont M, Barratt EM, Gottelli D, Kitchener AC, Daniels MJ, Pritchard JK et al (2001). Genetic diversity and introgression in the Scottish wildcat. Mol Ecol 10: 319-336.

Besansky NJ, Krzywinski J, Lehmann T, Simard F, Kern M, Mukabayire O et al (2003). Semiperrmeable species boundaries between Anopheles gambiae and Anopheles arabiensis: evidence from multilocus DNA sequence variation. Proc Natl Acc USA 100: 10818-10823.

Besansky NJ, Lehmann T, Fahey GT, Fontenille D, Braak LEO, Hawley WA et al (1997). Patterns of mitochondrial variation within and between African malaria vectors, Anopheles gambiae and $A$. arabiensis, suggest extensive gene flow. Genetics 147: 1817-1828.

Besansky NJ, Powell JR, Caccone A, Hamm DH, Scott JA, Collins FH (1994). Molecular phylogeny of the Anopheles gambiae suggests genetic introgression between the principle malaria vectors. Proc Natl Acad Sci USA 91: 6885-6888.

Caccone A, García BA, Powell JR (1996). Evolution of the mitochondrial DNA control region in the Anopheles gambiae complex. Insect Mol Biol 5: 51-59.

Charlwood JD, Vij R, Billingsley PF (2000). Dry season refugia of malaria-transmitting mosquitoes in a dry savannah zone of east Africa. Am J Trop Med Hyg 62: 726-732.

Clement M, Posada D, Crandall KA (2000). TCS: a computer program to estimate gene genealogies. Mol Ecol 9: 1657-1659.

Coluzzi M (1982). Spatial distribution of chromosomal inversions and speciation in anopheline mosquitoes. In: Bargozzi C (eds) Mechanisms of Speciation, Alan R. Liss: New York pp 143-153.

Crandall KA, Templeton AR (1993). Empirical tests of some predictions from coalescent theory with applications to intraspecific phylogeny reconstruction. Genetics 134: 959-969.

Della Torre A, Merzagora L, Powell JR, Coluzzi M (1997). Selective introgression of paracentric inversions between two sibling species of the Anopheles gambiae complex. Genetics $\mathbf{1 4 6}$ 239-244.

Donnelly MJ, Cuamba N, Charlwood JD, Collins FH, Townson $H$ (1999). Population structure in the malaria vector, Anopheles arabiensis Patton, in East Africa. Heredity 83: 408-417.

Donnelly MJ, Licht MC, Lehmann T (2001). Evidence for recent population expansion in the evolutionary history of the malaria vectors Anopheles arabiensis and Anopheles gambiae. Mol Biol Evol 18: 1353-1364.

Donnelly MJ, Simard F, Lehmann T (2002). Evolutionary studies of malaria vectors. Trends Parasitol 18: 75-80.

Donnelly MJ, Townson H (2000). Evidence for extensive genetic differentiation among populations of the malaria vector Anopheles arabiensis in eastern Africa. Insect Mol Biol 9: 357-367.

Evans BJ, Supriatna J, Melnick DJ (2001). Hybridization and population genetics of two macaque species in Sulawesi, Indonesia. Evolution 55: 1686-1702.

Favia G, Della Torre A, Bagayoko M, Lanfrancotti A, Sagnon NF, Touré YT et al (1997). Molecular identification of sympatric chromosomal forms of Anopheles gambiae and further evidence of their reproductive isolation. Insect $\mathrm{Mol}$ Biol 6: 377-383.

Gentile G, Slotman M, Ketmaier V, Powell JR, Caccone A (2001). Attempts to molecularly distinguish cryptic taxa in Anopheles gambiae s.s. Insect Mol Biol 10: 25-32. 
Goodman SJ, Barton NH, Swanson G, Abernethy K, Pemberton JM (1999). Introgression through rare hybridization: a genetic study of a hybrid zone between red and sika deer (genus Cervus) in Argyll, Scotland. Genetics 152: 355-371.

Grant PR, Grant BR (2002). Unpredictable evolution in a 30-year study of Darwin's finches. Science 296: 707-711.

Hudson RR, Boos DD, Kaplan NL (1992). Statistical test for detecting geographic subdivision. Mol Biol Evol 9: 138-151.

Kamau L, Lehmann T, Hawley WA, Orago ASS, Collins FH (1998). Microgeographic genetic differentiation of Anopheles gambiae mosquitoes from Asembo Bay, Western Kenya: a comparison with Kilifi in coastal Kenya. Am J Trop Med Hyg 58: 64-69.

Krzywinski J, Wilkerson RC, Besansky NJ (2001). Evolution of mitochondrial and ribosomal gene sequences in Anophelinae (Diptera:Culicidae): implications for phylogeny reconstruction. Mol Phylo Evol 18: 479-487.

Kumar S, Tamura K, Jakobsen IB, Nei M (2001). MEGA2: molecular evolutionary genetics analysis software. Bioinformatics 17: 1244-1245.

Lanzaro GC, Toure YT, Carnahan J, Zheng L, Dolo G, Traoré S et al (1998). Complexities in the genetic structure of Anopheles gambiae populations in west Africa as revealed by microsatellite DNA analysis. Proc Natl Acad Sci USA 95: 1426014265.

Lehmann T, Besansky NJ, Hawley WA, Fahey TG, Kamau L, Collins FH (1997). Microgeographic structure of Anopheles gambiae in western Kenya based on mtDNA and microsatellite loci. Mol Ecol 6: 243-253.

Lehmann T, Blackston CR, Besansky NJ, Escalante AA, Collins FH, Hawley WA (2000). The effect of the Rift Valley on gene flow between Anopheles gambiae populations in Kenya: the mtDNA perspective. J Hered 91: 165-168.

Lehmann T, Hawley WA, Grebert H, Collins FH (1998). The effective population size of Anopheles gambiae in Kenya: implications for population structure. Mol Biol Evol 15: 264-276.

Lehmann T, Licht MC, Elissa N, Maega BTA, Chimumbwa JM, Watsenga FT et al (2003). Population structure of Anopheles gambiae in Africa. J Hered 94: 133-147.

Martinsen GD, Whitham TG, Turek RJ, Keim P (2001). Hybrid populations selectively filter gene introgression between species. Evolution 55: 1325-1335.

Petrarca V, Beier JC (1992). Intraspecific chromosomal polymorphism in the Anopheles gambiae complex as a factor affecting malaria transmission in the Kisumu area of Kenya. Am J Trop Med Hyg 46: 229-237.

Pinto J, Donnelly MJ, Malta-Vacas J, Sousa CA, Gil V, Ferreira C et al (2003). An island within an island: genetic differentiation of Anopheles gambiae in São Tomé, West Africa, and its relevance to malaria vector control. Heredity 91: 407-414.
Pinto J, Donnelly MJ, Sousa CA, Gil V, Ferreira C, Elissa N et al (2002). Genetic structure of Anopheles gambiae (Diptera: Culicidae) in São Tomé and Príncipe (West Africa): implications for malaria control. Mol Ecol 11: 2183-2187.

Powell JR, Petrarca V, Della Torre A, Merzagora L, Caccone A, Coluzzi M (1999). Population structure, speciation and introgression in the Anopheles gambiae complex. Parassitologia 41: $101-114$

Roques S, Sevigny JM, Bernatchez L (2001). Evidence for broadscale introgressive hybridization between two redfish (genus Sebastes) in the North-west Atlantic: a rare marine example. Mol Ecol 10: 149-165.

SAS Institute Inc (1990). SAS Language: References, Version 6, 1st edn. Cary, NC.

Scott JA, Brogdon WG, Collins FH (1993). Identification of single specimens of the Anopheles gambiae complex by the polymerase chain reaction. Am J Trop Med Hyg 49: 520-529.

Simard F, Fontenille D, Lehmann T, Girod R, Brutus L, Gopaul $\mathrm{R}$ et al (1999). High amounts of genetic differentiation between populations of the malaria vector Anopheles arabiensis from west Africa and eastern outer islands. Am J Trop Med Hyg 60: 1000-1009.

Spiers AA, Mzilahowa T, Atkinson D, McCall PJ (2002). The malaria vectors of the Lower Shire Valley, Malawi. Malawi Med J 14: 4-7.

Templeton AR, Crandall KA, Sing CF (1992). A cladisticanalysis of phenotypic associations with haplotypes inferred from restriction endonuclease mapping and DNA-sequence data III Cladogram estimation. Genetics 132: 619-633.

Thelwell NJ, Huisman RA, Harbach RE, Butlin RK (2000). Evidence for mitochondrial introgression between Anopheles bwambae and Anopheles gambiae. Insect Mol Biol 9: 203-210.

Tripet F, Toure YT, Taylor CE, Norris DE, Dolo G, Lanzaro GC (2001). DNA analysis of transferred sperm reveals significant levels of gene flow between molecular forms of Anopheles gambiae. Mol Ecol 10: 1725-1732.

Walton C, Handley JM, Tun-Lin W, Collins FH, Harbach RE, Baimai V et al (2000). Population structure and population history of Anopheles dirus mosquitoes in southeast Asia. Mol Biol Evol 17: 962-974.

White GB, Magayuka SA, Boreham PFL (1972). Comparative studies on sibling species of the Anopheles gambiae Giles complex (Dipt., Culicidae): bionomics and vectorial activity of species A and species B at Segera, Tanzania. Bull Ent Res 62: 295-317.

Wondji C, Simard F, Fontenille D (2002). Evidence for genetic differentiation between the molecular forms $\mathrm{M}$ and $\mathrm{S}$ within the Forest chromosomal form of Anopheles gambiae in an area of sympatry. Insect Mol Biol 11: 11-19. 